Acta Crystallographica Section E

Structure Reports

Online

ISSN 1600-5368

\title{
cyclo-Tetrakis $\left(\mu_{2}\right.$-3-sulfidopropyl-
} $\left.\kappa^{3} C^{1}, S: S\right)$ tetrakis[chloridocobalt(III)]. Retraction

\section{Shafique Ahmad Awan, ${ }^{a}$ M. Nawaz Tahir, ${ }^{\text {b* }}$ Iram Khushi Muhammad," Saeed Ahmad ${ }^{c}$ and Muhammad Ilyas Tariq ${ }^{d}$}

${ }^{\mathbf{a}}$ PAEC, PO Box No. 1114, Islamabad GPO 44000, Pakistan, ${ }^{\mathbf{b}}$ Department of Physics, University of Sargodha, Sargodha, Pakistan, ${ }^{\mathbf{c}}$ Department of Chemistry, University of Engineering and Technology, Lahore 54890, Pakistan, and Department of Chemistry, University of Sargodha, Sargodha, Pakistan

Correspondence e-mail: dmntahir_uos@yahoo.com

Received 18 May 2012; accepted 18 May 2012

The paper by Awan et al. [Acta Cryst. (2011), E67, m576$\mathrm{m} 577]$ is retracted. It has subsequently been shown that the crystal studied was an already known zinc compound, $\left[\mathrm{Zn}_{4} \mathrm{Cl}_{4}\left(\mathrm{C}_{2} \mathrm{H}_{6} \mathrm{NS}\right)_{4}\right]$.

We wish to withdraw the paper by Awan et al. (2011) on a compound reported to be $\left[\mathrm{Co}_{4} \mathrm{Cl}_{4}\left(\mathrm{C}_{3} \mathrm{H}_{6} \mathrm{~S}\right)_{4}\right]$. It has subsequently been shown that the crystal studied was a related zinc compound, $\left[\mathrm{Zn}_{4} \mathrm{Cl}_{4}\left(\mathrm{C}_{2} \mathrm{H}_{6} \mathrm{NS}\right)_{4}\right]$, which was reported by Fleischer et al. (2006).

\section{References}

Awan, S. A., Tahir, M. N., Muhammad, I. K., Ahmad, S. \& Tariq, M. I. (2011). Acta Cryst. E67, m576-m577.

Fleischer, H., Hardt, S. \& Schollmeyer, D. (2006). Inorg. Chem. 45, 8318-8325. 
Acta Crystallographica Section E

\section{Structure Reports}

Online

ISSN 1600-5368

\section{cyclo-Tetrakis $\left(\mu_{2}\right.$-3-sulfidopropyl- $\left.\kappa^{3} C^{1}, S: S\right)$ tetrakis[chloridocobalt(III)]}

\section{Shafique Ahmad Awan, ${ }^{a}$ M. Nawaz Tahir, ${ }^{\text {b* }}$ Iram Khushi Muhammad, ${ }^{\mathrm{C}}$ Saeed Ahmad $^{\mathrm{c}}$ and Muhammad Ilyas Tariq ${ }^{\mathrm{d}}$}

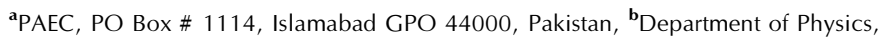
University of Sargodha, Sargodha, Pakistan, ${ }^{\mathbf{C}}$ Department of Chemistry, University of Engineering and Technology, Lahore 54890, Pakistan, and d Department of

Chemistry, University of Sargodha, Sargodha, Pakistan

Correspondence e-mail:dmntahir_uos@yahoo.com

Received 2 April 2011; accepted 7 April 2011

Key indicators: single-crystal X-ray study; $T=296 \mathrm{~K}$; mean $\sigma(\mathrm{C}-\mathrm{C})=0.011 \AA$; $R$ factor $=0.041 ; w R$ factor $=0.125 ;$ data-to-parameter ratio $=19.7$.

In the centrosymmetric title compound, $\left.\left[\mathrm{Co}_{4} \mathrm{Cl}_{4}\left(\mathrm{C}_{3} \mathrm{H}_{6} \mathrm{~S}\right)\right]_{4}\right]$, the two independent $\mathrm{Co}^{\mathrm{III}}$ ions are each coordinated in a distorted tetrahedral geometry by one $\mathrm{C}$, one $\mathrm{Cl}$ and two $\mathrm{S}$ atoms. The molecules are stabilized by $\mathrm{C}-\mathrm{H} \cdots \mathrm{Cl}$ hydrogen bonds. In the crystal, intermolecular $\mathrm{C}-\mathrm{H} \cdots \mathrm{Cl}$ and $\mathrm{C}-\mathrm{H} \cdots \mathrm{S}$ hydrogen bonds with $R_{2}^{2}(8), R_{4}^{2}(8)$ and $R_{2}^{2}(6)$ ring motifs generate a polymeric network.

\section{Related literature}

For related background see: Shahid et al. (2009); Altaf et al. (2010). For related structures, see: Duan et al. (1997); Tremel et al. (1992). For graph-set notation, see: Bernstein et al. (1995).

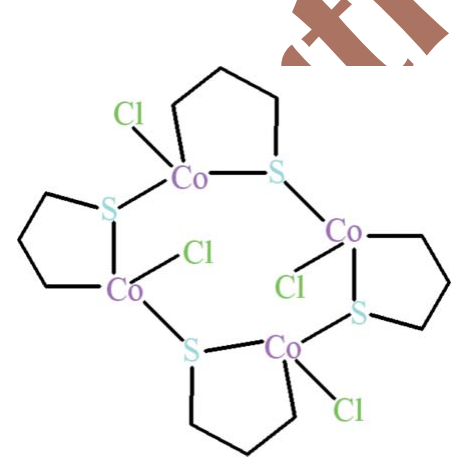

\section{Experimental}

\section{Crystal data}

$\left.\left[\mathrm{Co}_{4} \mathrm{Cl}_{4}\left(\mathrm{C}_{3} \mathrm{H}_{6} \mathrm{~S}\right)\right]_{4}\right]$

$M_{r}=674.07$

Monoclinic, $C 2 / c$

$a=23.6135(12) \AA$

$b=7.8465(3) \AA$

$c=16.8693(9) \AA$

$\beta=130.440(4)^{\circ}$

\section{Data collection}

Bruker Kappa APEXII CCD diffractometer

Absorption correction: multi-scan (SADABS; Bruker, 2005)

$T_{\min }=0.675, T_{\max }=0.683$

Refinement

$R\left[F^{2}>2 \sigma\left(F^{2}\right)\right]=0.041$

$w R\left(F^{2}\right)=0.125$

$S=1.04$

2152 reflections

13736 measured reflections 2152 independent reflections 1782 reflections with $I>2 \sigma(I)$ $R_{\text {int }}=0.058$

Table 1

Selected bond lengths $(\AA)$.

\begin{tabular}{llll}
\hline co1-Cl1 & $2.228(2)$ & $\mathrm{Co} 2-\mathrm{S} 1$ & $2.305(2)$ \\
$\mathrm{Co} 1-\mathrm{S} 1$ & $2.3570(17)$ & $\mathrm{Co} 2-\mathrm{S}^{\mathrm{i}}$ & $2.3648(16)$ \\
$\mathrm{Co} 1-\mathrm{S} 2$ & $2.318(2)$ & $\mathrm{Co} 2-\mathrm{C}^{\mathrm{i}}$ & $2.051(6)$ \\
$\mathrm{Co} 1-\mathrm{C} 3$ & $2.038(6)$ & $\mathrm{S} 1-\mathrm{C} 1$ & $1.826(7)$ \\
$\mathrm{Co} 2-\mathrm{Cl} 2$ & $2.236(3)$ & $\mathrm{S} 2-\mathrm{C} 4$ & $1.837(8)$ \\
\hline Symmetry code: (i) $-x+1$ & $y-1$ & &
\end{tabular}

Symmetry code: (i) $-x+\frac{1}{2}$

Table 2

Hydrogen-bond geometry $\left(\AA{ }^{\circ}\right)$.

\begin{tabular}{lllll}
\hline$D-\mathrm{H} \cdots A$ & $D-\mathrm{H}$ & $\mathrm{H} \cdots A$ & $D \cdots A$ & $D-\mathrm{H} \cdots A$ \\
\hline $\mathrm{C} 3-\mathrm{H} 3 A \cdots \mathrm{S}^{\mathrm{ii}}$ & 0.97 & 2.57 & $3.539(8)$ & 175 \\
$\mathrm{C} 3-\mathrm{H} 3 B \cdots \mathrm{Cl} 2^{\mathrm{iii}}$ & 0.97 & 2.74 & $3.512(7)$ & 138 \\
$\mathrm{C} 3-\mathrm{H} 3 B \cdots \mathrm{Cl} 2^{\mathrm{iv}}$ & 0.97 & 2.81 & $3.412(6)$ & 121 \\
$\mathrm{C} 6-\mathrm{H} 6 A \cdots \mathrm{Cl}^{\mathrm{i}}$ & 0.97 & 2.62 & $3.379(7)$ & 135 \\
$\mathrm{C} 6-\mathrm{H} 6 B \cdots \mathrm{Cl} 1^{\mathrm{v}}$ & 0.97 & 2.48 & $3.343(7)$ & 148 \\
\hline
\end{tabular}

Symmetry codes: (i) $-x+\frac{1}{2},-y-\frac{1}{2},-z+1$; (ii) $-x+\frac{1}{2}, y+\frac{1}{2},-z+\frac{1}{2}$; (iii) $-x, y,-z+\frac{1}{2}$; (iv) $x,-y, z-\frac{1}{2}$; (v) $x+\frac{1}{2},-y-\frac{1}{2}, z+\frac{1}{2}$.

Data collection: APEX2 (Bruker, 2009); cell refinement: SAINT (Bruker, 2009); data reduction: $S A I N T$; $\operatorname{program}(\mathrm{s})$ used to solve structure: SHELXS97 (Sheldrick, 2008); program(s) used to refine structure: SHELXL97 (Sheldrick, 2008); molecular graphics: ORTEPIII (Burnett \& Johnson, 1996), ORTEP-3 for Windows (Farrugia, 1997) and PLATON (Spek, 2009); software used to prepare material for publication: WinGX (Farrugia, 1999) and PLATON.

The authors acknowledge the provision of funds for the purchase of the diffractometer and encouragement by Dr Muhammad Akram Chaudhary, Vice Chancellor, University of Sargodha, Pakistan.

Supplementary data and figures for this paper are available from the IUCr electronic archives (Reference: SI2349).

\section{References}

Altaf, M., Stoeckli-Evans, H., Batool, S. S., Isab, A. A., Ahmad, S., Saleem, M., Awan, S. A. \& Shaheen, M. A. (2010). J. Coord. Chem. 63, 1176-1185.

Bernstein, J., Davis, R. E., Shimoni, L. \& Chang, N.-L. (1995). Angew. Chem. Int. Ed. Engl. 34, 1555-1573.

Bruker (2005). SADABS. Bruker AXS Inc., Madison, Wisconsin, USA.

Bruker (2009). APEX2 and SAINT. Bruker AXS Inc., Madison, Wisconsin, USA.

Burnett, M. N. \& Johnson, C. K. (1996). ORTEPIII. Report ORNL-6895. Oak Ridge National Laboratory, Tennessee, USA. 


\section{metal-organic compounds}

Duan, C.-Y., Liu, Z.-H., You, X.-Z., Xue, F. \& Mak, T. C. W. (1997). Chem. Commun. pp. 381-382.

Farrugia, L. J. (1997). J. Appl. Cryst. 30, 565.

Farrugia, L. J. (1999). J. Appl. Cryst. 32, 837-838.

Shahid, M., Rüffer, T., Lang, H., Awan, S. A. \& Ahmad, S. (2009). J. Coord. Chem. 62, 440-445.
Sheldrick, G. M. (2008). Acta Cryst. A64, 112-122.

Spek, A. L. (2009). Acta Cryst. D65, 148-155.

Tremel, W., Krebs, B., Greiwe, K., Simon, W., Stephan, H.-O. \& Henkel, G. (1992). Z. Naturforsch. Teil B, 47, 1580-1592.






\section{supporting information}

Acta Cryst. (2011). E67, m576-m577［doi:10.1107/S1600536811013067]

\section{cyclo-Tetrakis $\left(\mu_{2}-3\right.$-sulfidopropyl- $\left.\kappa^{3} C^{1}, S: S\right)$ tetrakis[chloridocobalt(III)]}

\section{Shafique Ahmad Awan, M. Nawaz Tahir, Iram Khushi Muhammad, Saeed Ahmad and Muhammad Ilyas Tariq}

\section{S1. Comment}

Recently, we have reported the crystal structure of zinc(II) and mercury(II) complexes of pyrrolidinedithiocarbamate (PDTC) (Shahid et al., 2009) \& (Altaf, et al., 2010). In the present study, we attempted to prepare a cobalt(II) complex with PDTC, but surprisingly the title compound (I, Fig. 1) was isolated, the crystal structure of which is being presented.

The crystal structure of (II) i.e, tetranuclear molecular square $[\mathrm{Co}(\mathrm{HL})]_{4}{ }^{4+}\left[\mathrm{H}_{2} \mathrm{~L}=\right.$ tetra(2-pyridyl)thiocarbazone] (Duan et al., 1997) and (III) i.e., bis(tetraethylammonium) hexakis( $\mu_{2}$-phenylthiolato)-tetrachloro-tetra -cobalt acetonitrile solvate (Tremel et al., 1992) have been published which are related to the fitle compound (I).

The crystal structure of the title compound (I) is centrosymmetric. The coordination around two independent $\mathrm{Co}^{\mathrm{iii}}$ ions is distorted tetrahedral from one $\mathrm{C}, \mathrm{Cl}$ and two $\mathrm{S}$-atoms. The range of $\mathrm{Co}-\mathrm{C}[2.038$ (6)-2.051 (6) $\AA]$ is shorter compared to $\mathrm{Co}-\mathrm{S}[2.305$ (2)-2.3648 (16) $\AA]$. The $\mathrm{Co}-\mathrm{Cl}$ bonds have values of 2.228 (2) and 2.236 (2) $\AA$. The important bond distances are given in Table 1. The molecules are stabilized in the form of a polymeric network due to $\mathrm{C}-\mathrm{H} \cdots \mathrm{Cl}$ and $\mathrm{C}-$ $\mathrm{H} \cdots \mathrm{S}$ intermolecular H-bonds (Table 2) forming $R_{2}{ }^{2}(8), R_{4}{ }^{2}(8)$ and $R_{2}{ }^{2}(6)$ ring motifs (Bernstein et al., 1995).

\section{S2. Experimental}

The title compound was prepared by adding two equivalents of ammonium pyrrolidinedithiocarbamate (PDTC) in $15 \mathrm{ml}$ methanol to a solution of $\mathrm{CoCl}_{2} \cdot 6 \mathrm{H}_{2} \mathrm{O}$ in $10 \mathrm{ml}$ methanol. The addition of PDTC in the pink colored metal ion solution resulted in the formation of green precipitates immediately. After stirring for half an hour, the precipitates were filtered off and dried. The blackish brown crystals of the title compound (I) were prepared by dissolving $0.03 \mathrm{~g}$ precipitates in 3 $\mathrm{ml}$ DMSO on heating in a vial and then cooling the resulting solution at room temperature.

\section{S3. Refinement}

The $\mathrm{H}$-atoms were positioned geometrically $(\mathrm{C}-\mathrm{H}=0.97 \AA)$ and were included in the refinement in the riding model approximation, with $U_{\text {iso }}(\mathrm{H})=x U_{\text {eq }}(\mathrm{C})$, where $x=1.2$ for all $\mathrm{H}$-atoms. 


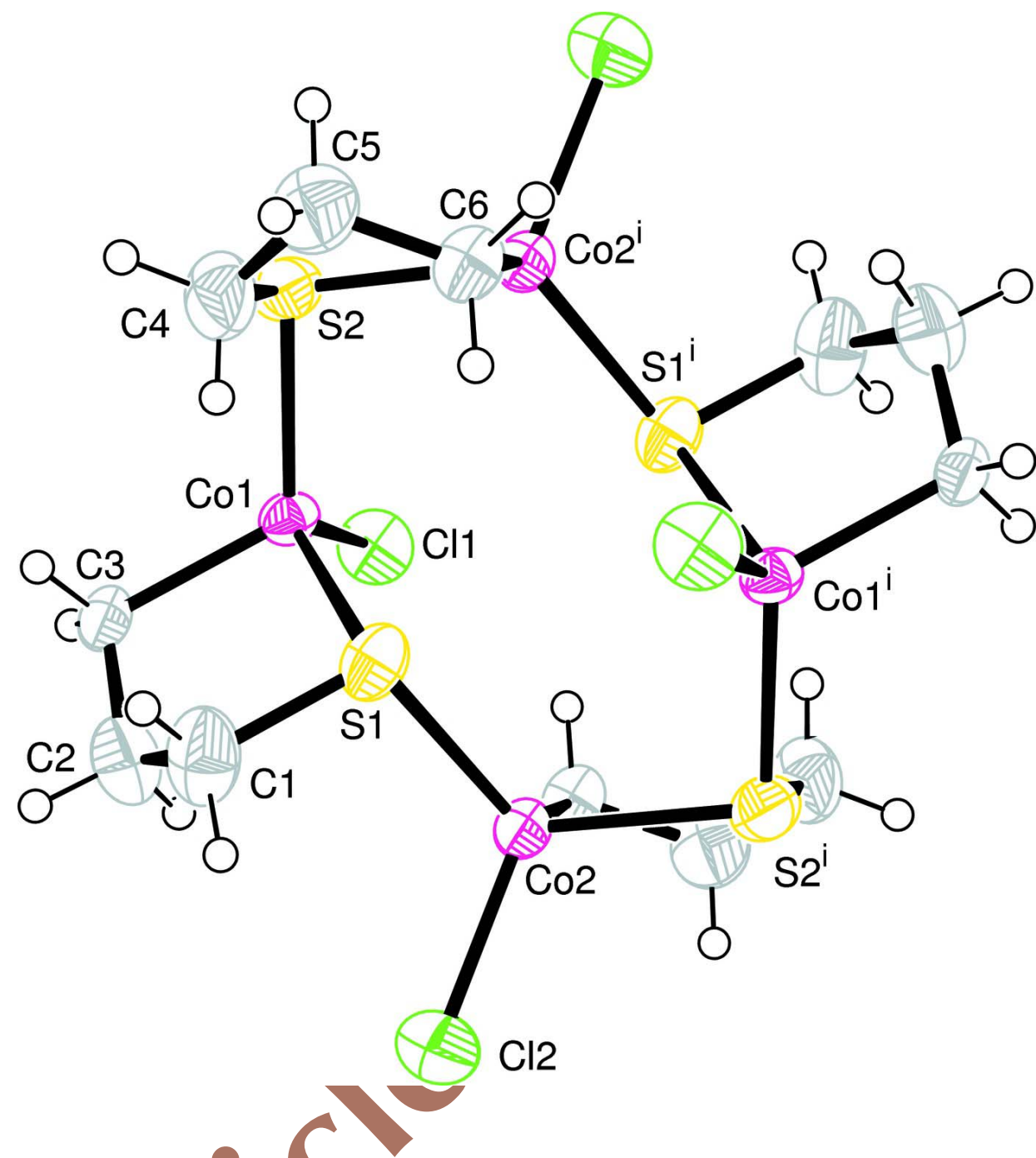

Figure 1

View of the centrosymmetric title compound. Symmetry code $i=-x+1 / 2,-y-1 / 2,-z+1$. The thermal ellipsoids are drawn at the $50 \%$ probability level. H-atoms are shown by small circles of arbitrary radii. 


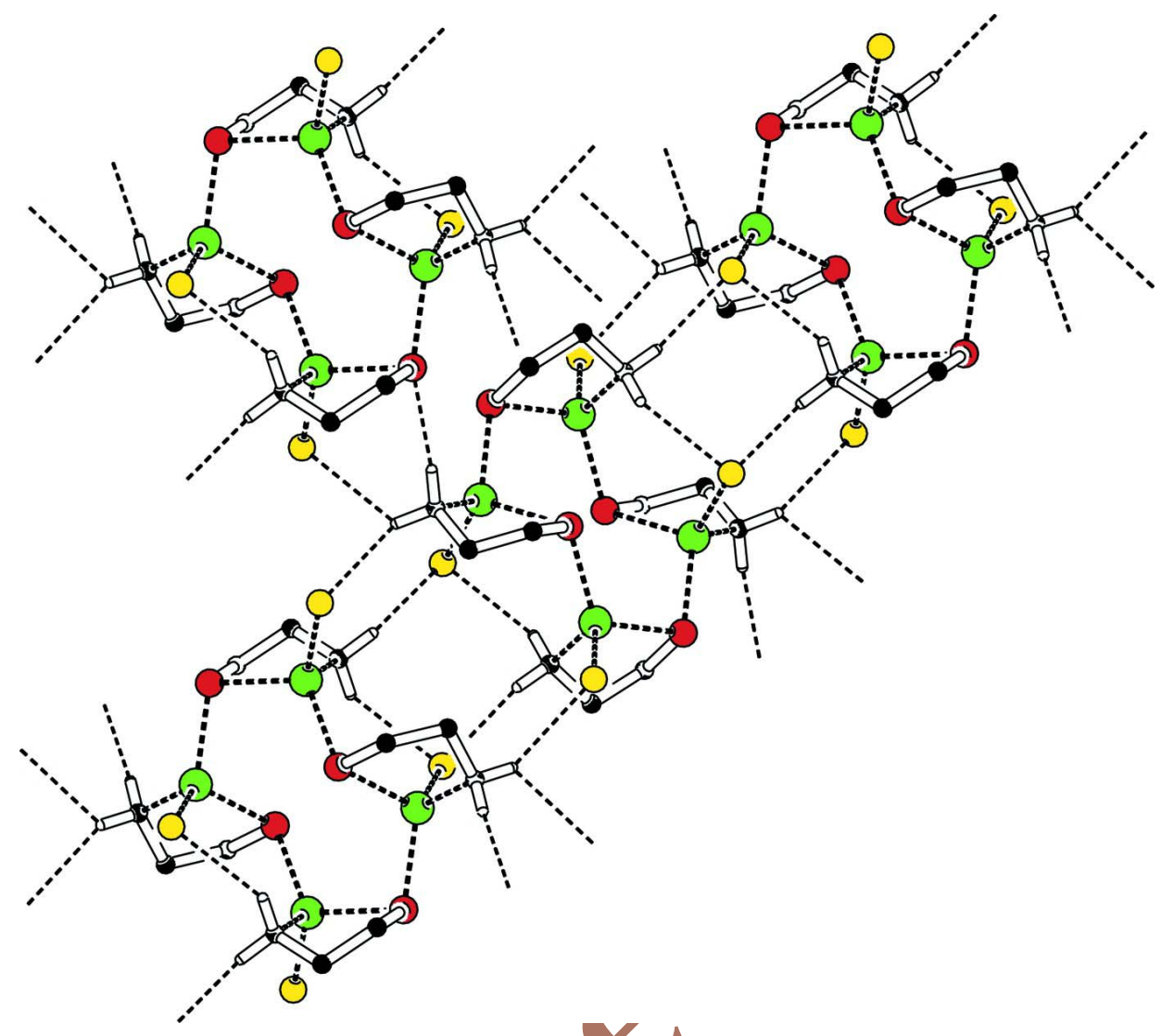

Figure 2

The partial packing (PLATON; Spek, 2009) which shows that molecules form a polymeric network with ring motifs. Hatoms not involved in H-bondings are omitted for clarity.

cyclo-Tetrakis $\left(\mu_{2}\right.$-3-sulfidopropyl- $\left.\kappa^{3} C^{1}, S: S\right)$ tetrakis[chloridocobalt(III)]

Crystal data

$\left.\left[\mathrm{Co}_{4} \mathrm{Cl}_{4}\left(\mathrm{C}_{3} \mathrm{H}_{6} \mathrm{~S}\right)\right]_{4}\right]$

$M_{r}=674.07$

Monoclinic, $C 2 / c$

Hall symbol: $-\mathrm{C} 2 \mathrm{yc}$

$a=23.6135(12) \AA$

$b=7.8465(3) \AA$

$c=16.8693(9) \AA$

$\beta=130.440(4)^{\circ}$

$V=2378.9(2) \AA^{3}$

$Z=4$

\section{Data collection}

Bruker Kappa APEXII CCD

diffractometer

Radiation source: fine-focus sealed tube

Graphite monochromator

Detector resolution: 8.10 pixels $\mathrm{mm}^{-1}$

$\omega$ scans

$$
F(000)=1344
$$

$D_{\mathrm{x}}=1.882 \mathrm{Mg} \mathrm{m}^{-3}$

Mo $K \alpha$ radiation, $\lambda=0.71073 \AA$

Cell parameters from 1782 reflections

$\theta=2.3-25.2^{\circ}$

$\mu=3.54 \mathrm{~mm}^{-1}$

$T=296 \mathrm{~K}$

Prisms, white

$0.24 \times 0.16 \times 0.14 \mathrm{~mm}$

Absorption correction: multi-scan

(SADABS; Bruker, 2005)

$T_{\min }=0.675, T_{\max }=0.683$

13736 measured reflections

2152 independent reflections

1782 reflections with $I>2 \sigma(I)$

$R_{\text {int }}=0.058$ 
$\theta_{\text {max }}=25.2^{\circ}, \theta_{\text {min }}=2.3^{\circ}$

$h=-28 \rightarrow 28$

Refinement

Refinement on $F^{2}$

Least-squares matrix: full

$R\left[F^{2}>2 \sigma\left(F^{2}\right)\right]=0.041$

$w R\left(F^{2}\right)=0.125$

$S=1.04$

2152 reflections

109 parameters

0 restraints

Primary atom site location: structure-invariant direct methods $k=-9 \rightarrow 8$

$l=-20 \rightarrow 20$

Secondary atom site location: difference Fourier map

Hydrogen site location: inferred from neighbouring sites

$\mathrm{H}$-atom parameters constrained

$w=1 /\left[\sigma^{2}\left(F_{\mathrm{o}}{ }^{2}\right)+(0.0653 P)^{2}+15.5359 P\right]$ where $P=\left(F_{\mathrm{o}}^{2}+2 F_{\mathrm{c}}{ }^{2}\right) / 3$

$(\Delta / \sigma)_{\max }<0.001$

$\Delta \rho_{\max }=0.70 \mathrm{e} \AA^{-3}$

$\Delta \rho_{\min }=-0.62$ e $\AA^{-3}$

Special details

Geometry. Bond distances, angles etc. have been calculated using the rounded fractional coordinates. All su's are estimated from the variances of the (full) variance-covariance matrix. The cell e.s.d.'s are taken into account in the estimation of distances, angles and torsion angles

Refinement. Refinement of $F^{2}$ against ALL reflections. The weighted $R$-factor $w R$ and goodness of fit $S$ are based on $F^{2}$, conventional $R$-factors $R$ are based on $F$, with $F$ set to zero for negative $F^{2}$. The threshold expression of $F^{2}>\sigma\left(F^{2}\right)$ is used only for calculating $R$-factors(gt) etc. and is not relevant to the choice of reflections for refinement. $R$-factors based on $F^{2}$ are statistically about twice as large as those based on $F$, and $R$-factors based on ALL data will be even larger.

Fractional atomic coordinates and isotropic or equivalent isotropic displacement parameters $\left(\AA^{2}\right)$

\begin{tabular}{|c|c|c|c|c|}
\hline & $x$ & $y$ & & $U_{\text {iso }} * / U_{\text {eq }}$ \\
\hline Col & $0.17597(4)$ & $-0.17781(9)$ & $0.30876(5)$ & $0.0323(2)$ \\
\hline $\mathrm{Co} 2$ & 0.14629 (4) & $-0.12119(9)$ & $0.50575(5)$ & $0.0336(2)$ \\
\hline $\mathrm{Cl1}$ & $0.09923(9)$ & $-0,3988(2)$ & $0.25283(12)$ & $0.0555(5)$ \\
\hline $\mathrm{Cl} 2$ & 0.07469 (11) & $0.0860(3)$ & $0.49043(15)$ & $0.0694(7)$ \\
\hline $\mathrm{S} 1$ & $0.21157(8)$ & $-0.0272(2)$ & $0.45587(11)$ & $0.0460(4)$ \\
\hline $\mathrm{S} 2$ & $0.28224(8)$ & $-0.2358(2)$ & $0.33225(11)$ & $0.0481(5)$ \\
\hline $\mathrm{C} 1$ & $0.1631(4)$ & (8) & $0.3814(5)$ & $0.066(3)$ \\
\hline $\mathrm{C} 2$ & 0.0959 (4) & $0.1316(9)$ & $0.2708(5)$ & $0.061(2)$ \\
\hline $\mathrm{C} 3$ & 0.1163 & 0.0287 (7) & $0.2182(4)$ & $0.0362(17)$ \\
\hline $\mathrm{C} 4$ & $0.3356(4)$ & $-0.0372(9)$ & $0.3904(6)$ & $0.064(3)$ \\
\hline $\mathrm{C} 5$ & 0.4140 & $-0.0689(10)$ & $0.4862(6)$ & $0.065(3)$ \\
\hline C6 & 0.4164 & $-0.1622(7)$ & $0.5638(4)$ & 0.0417 (17) \\
\hline H1A & 0.14799 & 0.22914 & 0.41469 & $0.0787^{*}$ \\
\hline H1B & 0.19711 & 0.23811 & 0.38263 & $0.0787^{*}$ \\
\hline $\mathrm{H} 2 \mathrm{~A}$ & 0.05934 & 0.07031 & 0.26878 & $0.0733^{*}$ \\
\hline $\mathrm{H} 2 \mathrm{~B}$ & 0.07391 & 0.23855 & 0.23404 & $0.0733^{*}$ \\
\hline $\mathrm{H} 3 \mathrm{~A}$ & 0.14572 & 0.09690 & 0.20863 & $0.0435^{*}$ \\
\hline H3B & 0.07165 & -0.00820 & 0.15024 & $0.0435^{*}$ \\
\hline H4A & 0.33533 & 0.02242 & 0.33979 & $0.0769 *$ \\
\hline H4B & 0.31166 & 0.03559 & 0.40757 & $0.0769 *$ \\
\hline H5A & 0.43922 & -0.13460 & 0.46849 & $0.0783 *$ \\
\hline H5B & 0.43980 & 0.03894 & 0.51537 & $0.0783 *$ \\
\hline H6A & 0.39644 & -0.09200 & 0.58805 & $0.0502 *$ \\
\hline H6B & 0.46733 & -0.19168 & 0.62322 & $0.0502 *$ \\
\hline
\end{tabular}


Atomic displacement parameters $\left(\AA^{2}\right)$

\begin{tabular}{lllllll}
\hline & $U^{11}$ & $U^{22}$ & $U^{33}$ & $U^{12}$ & $U^{13}$ & $U^{23}$ \\
\hline Co1 & $0.0275(4)$ & $0.0399(4)$ & $0.0300(4)$ & $-0.0012(3)$ & $0.0189(3)$ & $-0.0025(3)$ \\
Co2 & $0.0338(4)$ & $0.0373(4)$ & $0.0312(4)$ & $-0.0014(3)$ & $0.0217(3)$ & $0.0004(3)$ \\
C11 & $0.0496(9)$ & $0.0537(9)$ & $0.0545(9)$ & $-0.0089(7)$ & $0.0299(8)$ & $-0.0081(7)$ \\
C12 & $0.0683(11)$ & $0.0733(11)$ & $0.0725(12)$ & $0.0219(9)$ & $0.0483(10)$ & $0.0114(9)$ \\
S1 & $0.0485(8)$ & $0.0520(8)$ & $0.0377(7)$ & $-0.0099(7)$ & $0.0281(7)$ & $-0.0045(6)$ \\
S2 & $0.0407(8)$ & $0.0667(10)$ & $0.0398(8)$ & $0.0055(7)$ & $0.0274(7)$ & $0.0030(7)$ \\
C1 & $0.091(5)$ & $0.045(4)$ & $0.054(4)$ & $-0.010(4)$ & $0.044(4)$ & $-0.004(3)$ \\
C2 & $0.066(4)$ & $0.047(4)$ & $0.056(4)$ & $0.003(3)$ & $0.033(4)$ & $0.008(3)$ \\
C3 & $0.034(3)$ & $0.045(3)$ & $0.030(3)$ & $-0.003(2)$ & $0.021(2)$ & $0.005(2)$ \\
C4 & $0.056(4)$ & $0.064(4)$ & $0.074(5)$ & $0.000(3)$ & $0.043(4)$ & $0.022(4)$ \\
C5 & $0.056(4)$ & $0.067(4)$ & $0.074(5)$ & $-0.010(3)$ & $0.043(4)$ & $0.003(4)$ \\
C6 & $0.038(3)$ & $0.047(3)$ & $0.037(3)$ & $-0.012(2)$ & $0.023(3)$ & $-0.005(2)$ \\
\hline
\end{tabular}

Geometric parameters $\left(\AA,{ }^{\circ}\right)$

\begin{tabular}{|c|c|c|c|}
\hline $\mathrm{Co} 1-\mathrm{Cl1}$ & $2.228(2)$ & $\mathrm{C} 5-\mathrm{C} 6$ & $1.469(11)$ \\
\hline Co1-S1 & $2.3570(17)$ & $\mathrm{C} 1-\mathrm{H} 1 \mathrm{~A}$ & 0.9700 \\
\hline $\mathrm{Co} 1-\mathrm{S} 2$ & $2.318(2)$ & $\mathrm{C} 1-\mathrm{H} 1 \mathrm{~B}$ & 0.9700 \\
\hline $\mathrm{Co} 1-\mathrm{C} 3$ & $2.038(6)$ & $\mathrm{C} 2-\mathrm{H} 2 \mathrm{~A}$ & 0.9700 \\
\hline $\mathrm{Co} 2-\mathrm{Cl} 2$ & $2.236(3)$ & $\mathrm{C} 2-\mathrm{H} 2 \mathrm{~B}$ & 0.9700 \\
\hline $\mathrm{Co} 2-\mathrm{S} 1$ & $2.305(2)$ & C $3-\mathrm{H} 3 \mathrm{~A}$ & 0.9700 \\
\hline $\mathrm{Co} 2-\mathrm{S} 2^{\mathrm{i}}$ & $2.3648(16)$ & $\mathrm{C} 3-\mathrm{H} 3 \mathrm{~B}$ & 0.9700 \\
\hline $\mathrm{Co} 2-\mathrm{C}^{\mathrm{i}}$ & $2.051(6)$ & $\mathrm{C} 4-\mathrm{H} 4 \mathrm{~A}$ & 0.9700 \\
\hline $\mathrm{S} 1-\mathrm{C} 1$ & $1.826(7)$ & $\mathrm{C} 4-\mathrm{H} 4 \mathrm{~B}$ & 0.9700 \\
\hline $\mathrm{S} 2-\mathrm{C} 4$ & $1.837(8)$ & $\mathrm{C} 5-\mathrm{H} 5 \mathrm{~A}$ & 0.9700 \\
\hline $\mathrm{C} 1-\mathrm{C} 2$ & $1.495(10$ & $\mathrm{C} 5-\mathrm{H} 5 \mathrm{~B}$ & 0.9700 \\
\hline $\mathrm{C} 2-\mathrm{C} 3$ & $1.492(11)$ & $\mathrm{C} 6-\mathrm{H} 6 \mathrm{~A}$ & 0.9700 \\
\hline $\mathrm{C} 4-\mathrm{C} 5$ & & $\mathrm{C} 6-\mathrm{H} 6 \mathrm{~B}$ & 0.9700 \\
\hline $\mathrm{Co} 1 \cdots \mathrm{H}_{4} \mathrm{~A}^{\mathrm{ii}}$ & & $\mathrm{S} 2 \cdots \mathrm{H} 4 \mathrm{~A}^{\mathrm{ii}}$ & 3.0500 \\
\hline $\mathrm{Cl} 1 \cdots \mathrm{C} 3$ & $3.473(6)$ & $\mathrm{C} 2 \cdots \mathrm{Cl} 2^{\mathrm{iv}}$ & $3.570(7)$ \\
\hline $\mathrm{Cl} 1 \cdots \mathrm{C} 6^{\mathrm{iii}}$ & $3.343(7)$ & $\mathrm{C} 3 \cdots \mathrm{Cl} 1$ & $3.473(6)$ \\
\hline $\mathrm{C} 11 \cdots \mathrm{C} 6^{\mathrm{i}}$ & $3.379(7)$ & $\mathrm{C} 3 \cdots \mathrm{S} 1$ & $3.103(5)$ \\
\hline $\mathrm{Cl} 2 \cdots \mathrm{C} 1$ & $3.623(11)$ & $\mathrm{C} 3 \cdots \mathrm{S} 2$ & $3.686(7)$ \\
\hline $\mathrm{Cl} 2 \cdots \mathrm{C} 3^{\text {iv }}$ & $3.512(7)$ & $\mathrm{C} 3 \cdots \mathrm{C} 1$ & $2.455(9)$ \\
\hline $\mathrm{Cl} 2 \cdots \mathrm{C} 6^{\mathrm{i}}$ & $3.492(6)$ & $\mathrm{C} 3 \cdots \mathrm{Cl} 2^{\mathrm{iv}}$ & $3.512(7)$ \\
\hline $\mathrm{Cl} 2 \cdots \mathrm{C} 3^{v}$ & $3.412(6)$ & $\mathrm{C} 3 \cdots \mathrm{Cl} 2^{\text {viii }}$ & $3.412(6)$ \\
\hline $\mathrm{Cl} 2 \cdots \mathrm{S} 1$ & $3.744(4)$ & $\mathrm{C} 3 \cdots \mathrm{S} 2^{\mathrm{ix}}$ & $3.539(8)$ \\
\hline $\mathrm{C} 12 \cdots \mathrm{C} 2^{\mathrm{iv}}$ & $3.570(7)$ & $\mathrm{C} 6{ }^{\cdots} \mathrm{Cl} 1^{\mathrm{i}}$ & $3.379(7)$ \\
\hline $\mathrm{Cl} 1 \cdots \mathrm{H}_{4} \mathrm{~A}^{\mathrm{ii}}$ & 2.8900 & $\mathrm{C} 6 \cdots \mathrm{Cl} 2^{\mathrm{i}}$ & $3.491(6)$ \\
\hline $\mathrm{C} 11 \cdots \mathrm{H} 2 \mathrm{~B}^{\mathrm{vi}}$ & 2.8800 & $\mathrm{C} 6 \cdots \mathrm{Cl1}^{\mathrm{x}}$ & $3.343(7)$ \\
\hline $\mathrm{C} 11 \cdots \mathrm{H}_{6} \mathrm{~A}^{\mathrm{i}}$ & 2.6200 & $\mathrm{H} 1 \mathrm{~A} \cdots \mathrm{Cl} 2$ & 2.9600 \\
\hline $\mathrm{C} 11 \cdots \mathrm{H}_{6} \mathrm{~B}^{\mathrm{iii}}$ & 2.4800 & $\mathrm{H} 1 \mathrm{~B} \cdots \mathrm{H} 3 \mathrm{~A}$ & 2.5900 \\
\hline $\mathrm{Cl} 2 \cdots \mathrm{H} 1 \mathrm{~A}$ & 2.9600 & $\mathrm{H} 1 \mathrm{~B} \cdots \mathrm{S} 1^{\mathrm{vii}}$ & 3.1000 \\
\hline $\mathrm{Cl} 2 \cdots \mathrm{H} 3 \mathrm{~B}^{\mathrm{v}}$ & 2.8100 & $\mathrm{H} 2 \mathrm{~A} \cdots \mathrm{H} 2 \mathrm{~A}^{\mathrm{iv}}$ & 2.4400 \\
\hline
\end{tabular}




\begin{tabular}{|c|c|c|c|}
\hline $\mathrm{Cl} 2 \cdots \mathrm{H}_{3} \mathrm{~B}^{\mathrm{iv}}$ & 2.7400 & $\mathrm{H} 2 \mathrm{~B} \cdots \mathrm{C} 11^{\mathrm{xi}}$ & 2.8800 \\
\hline $\mathrm{Cl} 2 \cdots \mathrm{H} 5 \mathrm{~B}^{\mathrm{vii}}$ & 2.9600 & $\mathrm{H} 3 \mathrm{~A} \cdots \mathrm{H} 1 \mathrm{~B}$ & 2.5900 \\
\hline $\mathrm{S} 1 \cdots \mathrm{Cl} 2$ & $3.744(4)$ & $\mathrm{H} 3 \mathrm{~A} \cdots \mathrm{S} 2^{\mathrm{ix}}$ & 2.5700 \\
\hline $\mathrm{S} 1 \cdots \mathrm{S} 2$ & $3.785(3)$ & $\mathrm{H} 3 \mathrm{~B} \cdots \mathrm{Cl} 2^{\mathrm{iv}}$ & 2.7400 \\
\hline $\mathrm{S} 1 \cdots \mathrm{C} 2$ & $2.774(7)$ & $\mathrm{H} 3 \mathrm{~B} \cdots \mathrm{Cl} 2^{\mathrm{viii}}$ & 2.8100 \\
\hline $\mathrm{S} 1 \cdots \mathrm{C} 3$ & $3.103(5)$ & $\mathrm{H} 4 \mathrm{~A} \cdots \mathrm{Co}^{\mathrm{ix}}$ & 3.3200 \\
\hline $\mathrm{S} 1 \cdots \mathrm{Co}^{\mathrm{i}}$ & $3.8074(16)$ & $\mathrm{H} 4 \mathrm{~A} \cdots \mathrm{Cl} 1^{\mathrm{ix}}$ & 2.8900 \\
\hline $\mathrm{S} 1 \cdots \mathrm{S} 1^{\mathrm{i}}$ & $3.773(2)$ & $\mathrm{H} 4 \mathrm{~A} \cdots \mathrm{S} 2^{\mathrm{ix}}$ & 3.0500 \\
\hline $\mathrm{S} 2 \cdots \mathrm{C} 3^{\mathrm{ii}}$ & $3.539(8)$ & $\mathrm{H} 4 \mathrm{~B} \cdots \mathrm{S} 1$ & 3.0000 \\
\hline $\mathrm{S} 2 \cdots \mathrm{S} 1$ & $3.785(3)$ & H4B $\cdots \mathrm{H} 6 \mathrm{~A}$ & 2.5200 \\
\hline $\mathrm{S} 2 \cdots \mathrm{C} 3$ & $3.686(7)$ & $\mathrm{H} 5 \mathrm{~B} \cdots \mathrm{Cl} 2^{\mathrm{vii}}$ & 2.9600 \\
\hline $\mathrm{S} 1 \cdots \mathrm{H} 4 \mathrm{~B}$ & 3.0000 & H6A $\cdots \mathrm{H} 4 \mathrm{~B}$ & 2.5200 \\
\hline $\mathrm{S} 1 \cdots \mathrm{H} 1 \mathrm{~B}^{\mathrm{vii}}$ & 3.1000 & $\mathrm{H} 6 \mathrm{~A} \cdots \mathrm{Cl} 1^{\mathrm{i}}$ & 2.6200 \\
\hline $\mathrm{S} 2 \cdots \mathrm{H} 3 \mathrm{~A}^{\mathrm{ii}}$ & 2.5700 & $\mathrm{H} 6 \mathrm{~B} \cdots \mathrm{C} 11^{\mathrm{x}}$ & \\
\hline $\mathrm{C} 11-\mathrm{Co} 1-\mathrm{S} 1$ & $118.22(8)$ & $\mathrm{C} 2-\mathrm{C} 1-\mathrm{H} 1 \mathrm{~B}$ & \\
\hline $\mathrm{C} 11-\mathrm{Co} 1-\mathrm{S} 2$ & $114.37(8)$ & $\mathrm{H} 1 \mathrm{~A}-\mathrm{C} 1-\mathrm{H} 1 \mathrm{~B}$ & 108.00 \\
\hline $\mathrm{C} 11-\mathrm{Co} 1-\mathrm{C} 3$ & $108.9(2)$ & $\mathrm{C} 1-\mathrm{C} 2-\mathrm{H} 2 \mathrm{~A}$ & 110.00 \\
\hline $\mathrm{S} 1-\mathrm{Co} 1-\mathrm{S} 2$ & $108.14(7)$ & $\mathrm{C} 1-\mathrm{C} 2-\mathrm{H} 2 \mathrm{~B}$ & 109.00 \\
\hline $\mathrm{S} 1-\mathrm{Co} 1-\mathrm{C} 3$ & $89.52(17)$ & $\mathrm{C} 3-\mathrm{C} 2-\mathrm{H} 2 \mathrm{~A}$ & 110.00 \\
\hline $\mathrm{S} 2-\mathrm{Co} 1-\mathrm{C} 3$ & $115.5(2)$ & $\mathrm{C} 3-\mathrm{C} 2$ & 109.00 \\
\hline $\mathrm{Cl} 2-\mathrm{Co} 2-\mathrm{S} 1$ & $111.09(9)$ & $-\mathrm{H} 2 \mathrm{~B}$ & 108.00 \\
\hline $\mathrm{Cl} 2-\mathrm{Co} 2-\mathrm{S} 2^{\mathrm{i}}$ & $113.44(8)$ & $3-\mathrm{H} 3 \mathrm{~A}$ & 110.00 \\
\hline $\mathrm{Cl} 2-\mathrm{Co} 2-\mathrm{C}^{\mathrm{i}}$ & $109.0(2)$ & $\mathrm{COl}-\mathrm{C} 3-\mathrm{H} 3 \mathrm{~B}$ & 110.00 \\
\hline $\mathrm{S} 1-\mathrm{Co} 2-\mathrm{S} 2^{\mathrm{i}}$ & $115.39(8)$ & $-\mathrm{H} 3 \mathrm{~A}$ & 110.00 \\
\hline $\mathrm{S} 1-\mathrm{Co} 2-\mathrm{C} 6^{\mathrm{i}}$ & $117.5(2)$ & $\mathrm{C} 2-\mathrm{C} 3-\mathrm{H} 3 \mathrm{~B}$ & 110.00 \\
\hline $\mathrm{S} 2^{\mathrm{i}}-\mathrm{Co} 2-\mathrm{C} 6^{\mathrm{i}}$ & $88.68(15)$ & $\mathrm{H} 3 \mathrm{~A}-\mathrm{C} 3-\mathrm{H} 3 \mathrm{~B}$ & 108.00 \\
\hline $\mathrm{Co} 1-\mathrm{S} 1-\mathrm{Co} 2$ & $110.96(8)$ & $\mathrm{S} 2-\mathrm{C} 4-\mathrm{H} 4 \mathrm{~A}$ & 109.00 \\
\hline $\mathrm{Co} 1-\mathrm{S} 1-\mathrm{C} 1$ & 93.3 & $\mathrm{~S} 2-\mathrm{C} 4-\mathrm{H} 4 \mathrm{~B}$ & 109.00 \\
\hline $\mathrm{Co} 2-\mathrm{S} 1-\mathrm{C} 1$ & $104.4(4)$ & $\mathrm{C} 5-\mathrm{C} 4-\mathrm{H} 4 \mathrm{~A}$ & 109.00 \\
\hline $\mathrm{Co} 1-\mathrm{S} 2-\mathrm{C} 4$ & $102.5(4)$ & $\mathrm{C} 5-\mathrm{C} 4-\mathrm{H} 4 \mathrm{~B}$ & 109.00 \\
\hline $\mathrm{Co} 1-\mathrm{S} 2-\mathrm{Co} 2^{\mathrm{i}}$ & & $\mathrm{H} 4 \mathrm{~A}-\mathrm{C} 4-\mathrm{H} 4 \mathrm{~B}$ & 108.00 \\
\hline $\mathrm{Co} 2 \mathrm{i}-\mathrm{S} 2-\mathrm{C} 4$ & (2) & $\mathrm{C} 4-\mathrm{C} 5-\mathrm{H} 5 \mathrm{~A}$ & 110.00 \\
\hline $\mathrm{S} 1-\mathrm{C} 1-\mathrm{C} 2$ & $113.0(5)$ & $\mathrm{C} 4-\mathrm{C} 5-\mathrm{H} 5 \mathrm{~B}$ & 110.00 \\
\hline $\mathrm{C} 1-\mathrm{C} 2-\mathrm{C} 3$ & $110.6(8)$ & $\mathrm{C} 6-\mathrm{C} 5-\mathrm{H} 5 \mathrm{~A}$ & 109.00 \\
\hline $\mathrm{Co} 1-\mathrm{C} 3-\mathrm{C} 2$ & $108.5(4)$ & $\mathrm{C} 6-\mathrm{C} 5-\mathrm{H} 5 \mathrm{~B}$ & 110.00 \\
\hline $\mathrm{S} 2-\mathrm{C} 4-\mathrm{C} 5$ & $112.1(5)$ & $\mathrm{H} 5 \mathrm{~A}-\mathrm{C} 5-\mathrm{H} 5 \mathrm{~B}$ & 108.00 \\
\hline $\mathrm{C} 4-\mathrm{C} 5-\mathrm{C} 6$ & $110.6(9)$ & $\mathrm{C} 5-\mathrm{C} 6-\mathrm{H} 6 \mathrm{~A}$ & 110.00 \\
\hline $\mathrm{Co} 2-\mathrm{C} 6-\mathrm{C} 5$ & $107.9(4)$ & $\mathrm{C} 5-\mathrm{C} 6-\mathrm{H} 6 \mathrm{~B}$ & 110.00 \\
\hline $\mathrm{S} 1-\mathrm{C} 1-\mathrm{H} 1 \mathrm{~A}$ & 109.00 & $\mathrm{H} 6 \mathrm{~A}-\mathrm{C} 6-\mathrm{H} 6 \mathrm{~B}$ & 108.00 \\
\hline $\mathrm{S} 1-\mathrm{C} 1-\mathrm{H} 1 \mathrm{~B}$ & 109.00 & $\mathrm{Co} 2-\mathrm{C} 6-\mathrm{H} 6 \mathrm{~A}$ & 110.00 \\
\hline $\mathrm{C} 2-\mathrm{C} 1-\mathrm{H} 1 \mathrm{~A}$ & 109.00 & $\mathrm{Co} 2-\mathrm{C} 6-\mathrm{H} 6 \mathrm{~B}$ & 110.00 \\
\hline $\mathrm{Cl} 1-\mathrm{Co} 1-\mathrm{S} 1-\mathrm{Co} 2$ & $-7.06(11)$ & $\mathrm{S} 22^{\mathrm{i}}-\mathrm{Co} 2-\mathrm{S} 1-\mathrm{C} 1$ & $-148.1(2)$ \\
\hline $\mathrm{C} 11-\mathrm{Co} 1-\mathrm{S} 1-\mathrm{C} 1$ & $-113.8(3)$ & $\mathrm{C} 6-\mathrm{Co} 2-\mathrm{S} 1-\mathrm{Co} 1$ & $10.1(2)$ \\
\hline $\mathrm{S} 2-\mathrm{Co} 1-\mathrm{S} 1-\mathrm{Co} 2$ & $-139.02(7)$ & $\mathrm{C} 6-\mathrm{Co} 2-\mathrm{S} 1-\mathrm{C} 1$ & $109.3(3)$ \\
\hline $\mathrm{S} 2-\mathrm{Co} 1-\mathrm{S} 1-\mathrm{C} 1$ & $114.2(3)$ & $\mathrm{Cl} 2-\mathrm{Co} 2-\mathrm{S} 2-\mathrm{Co}^{\mathrm{i}}$ & $-145.79(9)$ \\
\hline $\mathrm{C} 3-\mathrm{Co} 1-\mathrm{S} 1-\mathrm{Co} 2$ & $104.3(2)$ & $\mathrm{Cl} 2-\mathrm{Co} 2-\mathrm{S} 2^{\mathrm{i}}-\mathrm{C} 4^{\mathrm{i}}$ & $110.9(4)$ \\
\hline
\end{tabular}




\begin{tabular}{|c|c|c|c|}
\hline $\mathrm{C} 3-\mathrm{Co} 1-\mathrm{S} 1-\mathrm{C} 1$ & $-2.5(4)$ & $\mathrm{S} 1-\mathrm{Co} 2-\mathrm{S} 2^{\mathrm{i}}-\mathrm{Co}^{\mathrm{i}}$ & $-16.01(8)$ \\
\hline $\mathrm{Cl} 1-\mathrm{Co} 1-\mathrm{S} 2-\mathrm{C} 4$ & $-174.3(3)$ & $\mathrm{S} 1-\mathrm{Co} 2-\mathrm{S} 2^{\mathrm{i}}-\mathrm{C} 4^{\mathrm{i}}$ & $-119.3(4)$ \\
\hline $\mathrm{Cl} 1-\mathrm{Co} 1-\mathrm{S} 2-\mathrm{Co}^{\mathrm{i}}$ & $-78.66(7)$ & $\mathrm{Cl} 2-\mathrm{Co} 2-\mathrm{C}^{\mathrm{i}}-\mathrm{C} 5^{\mathrm{i}}$ & $-87.1(6)$ \\
\hline $\mathrm{S} 1-\mathrm{Co} 1-\mathrm{S} 2-\mathrm{C} 4$ & $-40.3(3)$ & $\mathrm{S} 1-\mathrm{Co} 2-\mathrm{C} 6^{\mathrm{i}}-\mathrm{C} 5^{\mathrm{i}}$ & $145.4(5)$ \\
\hline $\mathrm{S} 1-\mathrm{Co} 1-\mathrm{S} 2-\mathrm{Co} 2^{\mathrm{i}}$ & $55.34(7)$ & $\mathrm{Co} 1-\mathrm{S} 1-\mathrm{C} 1-\mathrm{C} 2$ & $30.3(8)$ \\
\hline $\mathrm{C} 3-\mathrm{Co} 1-\mathrm{S} 2-\mathrm{C} 4$ & $58.1(3)$ & $\mathrm{Co} 2-\mathrm{S} 1-\mathrm{C} 1-\mathrm{C} 2$ & $-82.3(7)$ \\
\hline $\mathrm{C} 3-\mathrm{Co} 1-\mathrm{S} 2-\mathrm{Co}^{\mathrm{i}}$ & $153.74(17)$ & $\mathrm{Co} 1-\mathrm{S} 2-\mathrm{C} 4-\mathrm{C} 5$ & $130.1(7)$ \\
\hline $\mathrm{C} 11-\mathrm{Co} 1-\mathrm{C} 3-\mathrm{C} 2$ & $95.3(5)$ & $\mathrm{Co} 2-\mathrm{S} 2-\mathrm{C} 4-\mathrm{C} 5$ & $29.2(7)$ \\
\hline $\mathrm{S} 1-\mathrm{Co} 1-\mathrm{C} 3-\mathrm{C} 2$ & $-24.5(5)$ & $\mathrm{S} 1-\mathrm{C} 1-\mathrm{C} 2-\mathrm{C} 3$ & $-56.3(9)$ \\
\hline $\mathrm{S} 2-\mathrm{Co} 1-\mathrm{C} 3-\mathrm{C} 2$ & $-134.5(5)$ & $\mathrm{C} 1-\mathrm{C} 2-\mathrm{C} 3-\mathrm{Co} 1$ & $51.0(7)$ \\
\hline $\mathrm{Cl} 2-\mathrm{Co} 2-\mathrm{S} 1-\mathrm{Co} 1$ & $-116.41(8)$ & $\mathrm{S} 2-\mathrm{C} 4-\mathrm{C} 5-\mathrm{C} 6$ & $-57.7(9)$ \\
\hline $\mathrm{Cl} 2-\mathrm{Co} 2-\mathrm{S} 1-\mathrm{C} 1$ & $-17.1(2)$ & $\mathrm{C} 4-\mathrm{C} 5-\mathrm{C} 6-\mathrm{Co}^{\mathrm{i}}$ & $54.4(7)$ \\
\hline $\mathrm{S} 2-\mathrm{Co} 2-\mathrm{S} 1-\mathrm{Co} 1$ & $112.67(7)$ & & \\
\hline \multirow{2}{*}{\multicolumn{4}{|c|}{$\begin{array}{l}\text { Symmetry codes: (i) }-x+1 / 2,-y-1 / 2,-z+1 \text {; (ii) }-x+1 / 2, y-1 / 2,-z+1 / 2 \text {; (iii) } x-1 / 2,-y-1 / 2, z-1 / 2 \text {; (iv) }-x, y,-z+1 / 2 \text {; (v) } x,-y, z+1 / 2 \text {; (vi) } x, y-1 \text {, } \\
-x+1 / 2,-y+1 / 2,-z+1 \text {; (viii) } x,-y, z-1 / 2 \text {; (ix) }-x+1 / 2, y+1 / 2,-z+1 / 2 \text {; (x) } x+1 / 2,-y-1 / 2, z+1 / 2 \text {; (xi) } x, y+1, z \text {. }\end{array}$}} \\
\hline & & & \\
\hline$D-\mathrm{H} \cdots A$ & $D-\mathrm{H}$ & $\mathrm{H} \cdots A$ & $D-\mathrm{H} \cdots A$ \\
\hline $\mathrm{C} 3-\mathrm{H} 3 A \cdots \mathrm{S} 2^{\mathrm{ix}}$ & 0.97 & 2.57 & 175 \\
\hline $\mathrm{C} 3-\mathrm{H} 3 B \cdots \mathrm{Cl} 2^{\mathrm{iv}}$ & 0.97 & & 138 \\
\hline $\mathrm{C} 3-\mathrm{H} 3 B^{\cdots \cdots} \mathrm{Cl} 2^{\text {viii }}$ & 0.97 & & 121 \\
\hline $\mathrm{C} 6-\mathrm{H} 6 A \cdots \mathrm{Cl} 1^{\mathrm{i}}$ & 0.97 & & 135 \\
\hline $\mathrm{C} 6-\mathrm{H} 6 B \cdots \mathrm{Cl1}{ }^{\mathrm{x}}$ & 0.97 & & 148 \\
\hline
\end{tabular}

Symmetry codes: (i) $-x+1 / 2,-y-1 / 2,-z+1$; (iv) $-x, y,-z+1 / 2$; (viii) $x,-y, z-1 / 2$; (ix) $-x+1 / 2, y+1 / 2,-z+1 / 2$; (x) $x+1 / 2,-y-1 / 2, z+1 / 2$. 